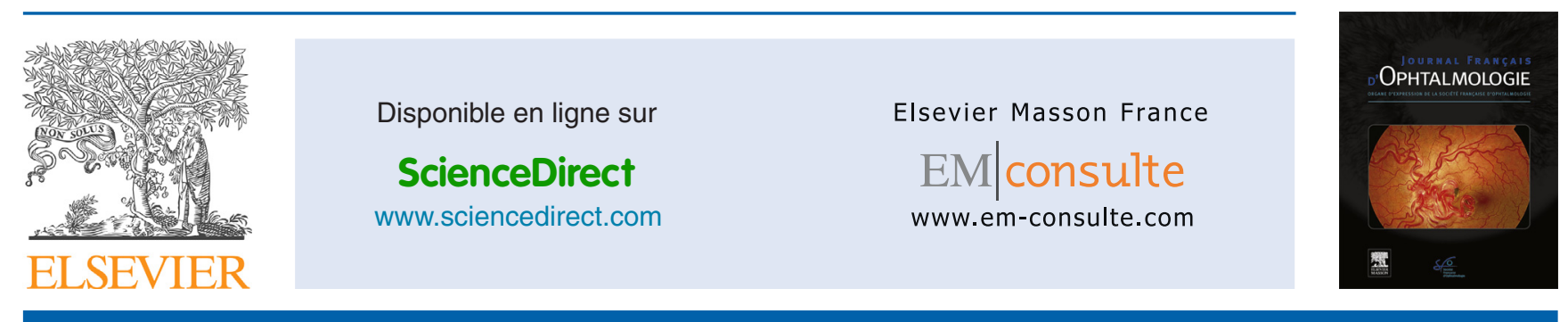

\title{
LETTRE À L’ÉDITEUR
}

\section{Conjonctivite à chlamydia trachomatis}

\section{Chlamydia trachomatis conjunctivitis}

Un homme de 22 ans se présente aux urgences ophtalmologiques pour la présence, depuis 3 semaines, d'un œil gauche rouge accompagné d'une sensation de corps étranger et de sécrétions collantes. L'acuité visuelle est chiffrée à 10/10 aux deux yeux avec une importante correction de myopie.

L'examen biomicroscopique met en évidence de nombreux follicules (hyperplasie du tissu lymphoïde de la conjonctive) situés au niveau de la conjonctive palpébrale, présents en plus grands nombres à gauche. Il n'y a pas d'atteinte cornéenne ni de signe d'uvéite. Un diagnostic de conjonctivite virale est posé et un traitement par De Icol $^{\circledR}$ collyre (chloramphénicol + dexaméthasone) est initié.

Cinq semaines plus tard, après deux mois d'évolution, il revient aux urgences. La situation s'est nettement aggravée avec des follicules géants (Fig. 1) et une adénopathie prétragienne à gauche.

Des prélèvements conjonctivaux sur écouvillons sont réalisés pour rechercher par PCR le Chlamydia Trachomatis (résultat positif, sans spécifier le sérotype) et le Neisseria Gonorrhea (résultat négatif). D'autres prélèvements sont faits au niveau de l'oropharynx, de l'anus et dans les urines. Le bilan est complété par des sérologies virales (VIH, hépatite $A / B / C$ ) et bactérienne (syphilis). Tous ces examens sont négatifs.

Un diagnostic de conjonctivite à Chlamydia Trachomatis est posé et un traitement par une prise unique d'azythromycine $1 \mathrm{~g}$ per os et de '’Azyter $^{\circledR}$ (azythromycine collyre à prendre $2 \times /$ jour durant 3 jours) est initié.

En deux semaines, on constate une amélioration qui est plus lente les mois suivants. De la cortisone topique est utilisée transitoirement pour diminuer les plaintes et la folliculose réactionnelle.

Nous totalisons 8 cas de conjonctivites à chlamydia en 6 mois dans notre service. Selon les statistiques nationales belges, les infections à chlamydia trachomatis sont en augmentation [1].

Différentes entités cliniques existent en fonction du sérotype. Les sérotypes A, B ou C causent le trachome, une conjonctivite à chlamydia sévissant dans les pays en voie de développement et entraînant des lésions cornéennes irréversibles. Les sérotypes $\mathrm{D}$ à $\mathrm{K}$ sont responsables de la conjonctivite du nouveau-né et de la conjonctivite folliculaire (à inclusion) de l'adulte qui est le cas nous concernant $[2,3]$.

Aucun élément n'est pathognomonique mais la conjonction d'éléments anamnestiques et cliniques rend le cas suspect et doit pousser le clinicien à réaliser une PCR [2,3]. Parmi ceux-ci, nous pouvons retenir, sur le plan anamnestique, une inefficacité du traitement classique, une errance diagnostique et le caractère chronique $[3,4]$. Sur le plan clinique, retenons le caractère unilatéral ou asymétrique et la présence de follicules géants [5].

Il s'agit d'une infection sexuellement transmissible. Il convient de s'assurer de l'absence de signes présents ou passés d'infection urinaire ou génitale $[5,6]$.

Le mode de transmission peut être variable $[2,3,7]$. La forme la plus classique est la transmission par les mains à partir de sécrétions génitales [7]. Une infection à chlamydia ne signifie pas forcément un rapport extra-conjugal. Il peut s'agir d'un portage parfois ancien de plusieurs années. La transmission d'œil à œil ou à partir de surfaces contaminées est également possible [7]. Enfin, la contamination, dans des piscines insuffisamment désinfectées, est possible [2].

Pour ce cas, ainsi que pour les autres cas rencontrés dans le service, aucune infection urinaire ou génitale n'a été retrouvée [7].

Le diagnostic différentiel est celui des conjonctivites folliculaires. La conjonctivite à adénovirus est la cause la plus fréquente d'œil rouge. Elle est aussi plus fulminante, hautement contagieuse (bilatéralisation rapide) et peut laisser des cicatrices cornéennes $[4,8]$. Une atteinte cornéenne de type kératite ponctuée superficielle ou infiltrats sous épithéliaux peut être associée pouvant alors mimer les numules retrouvées dans la conjonctivite virale classique à adénovirus [7]. L'herpès est à évoquer et à ne pas manquer, au vu de sa fréquence, et de ses complications potentiellement cécitantes. Le gonocoque donne des conjonctivites fulminantes en $24 \mathrm{~h}$ avec des sécrétions très purulentes. La réaction folliculaire peut être si importante en envahissant la conjonctive bulbaire périphérique qu'elle peut mimer le lymphome conjonctival.

Pour confirmer le diagnostic, nous avons utilisé la technique de PCR sur un écouvillon pour germes atypiques.

Le traitement est important pour limiter les conséquences systémiques (salpingo-ovarite, infertilité) et la contagion [3]. Le dépistage, pour d'autres IST (infections sexuellement transmissibles), pour le patient et ses partenaires sexuels, est recommandé [3]. La prise unique d'1 g d'azythromycine suffit $[3,7,9]$. La doxycycline, $100 \mathrm{mg}$ deux fois par jour durant 7 jours, est une alternative. Tous les partenaires sexuels doivent être traités [3]. 

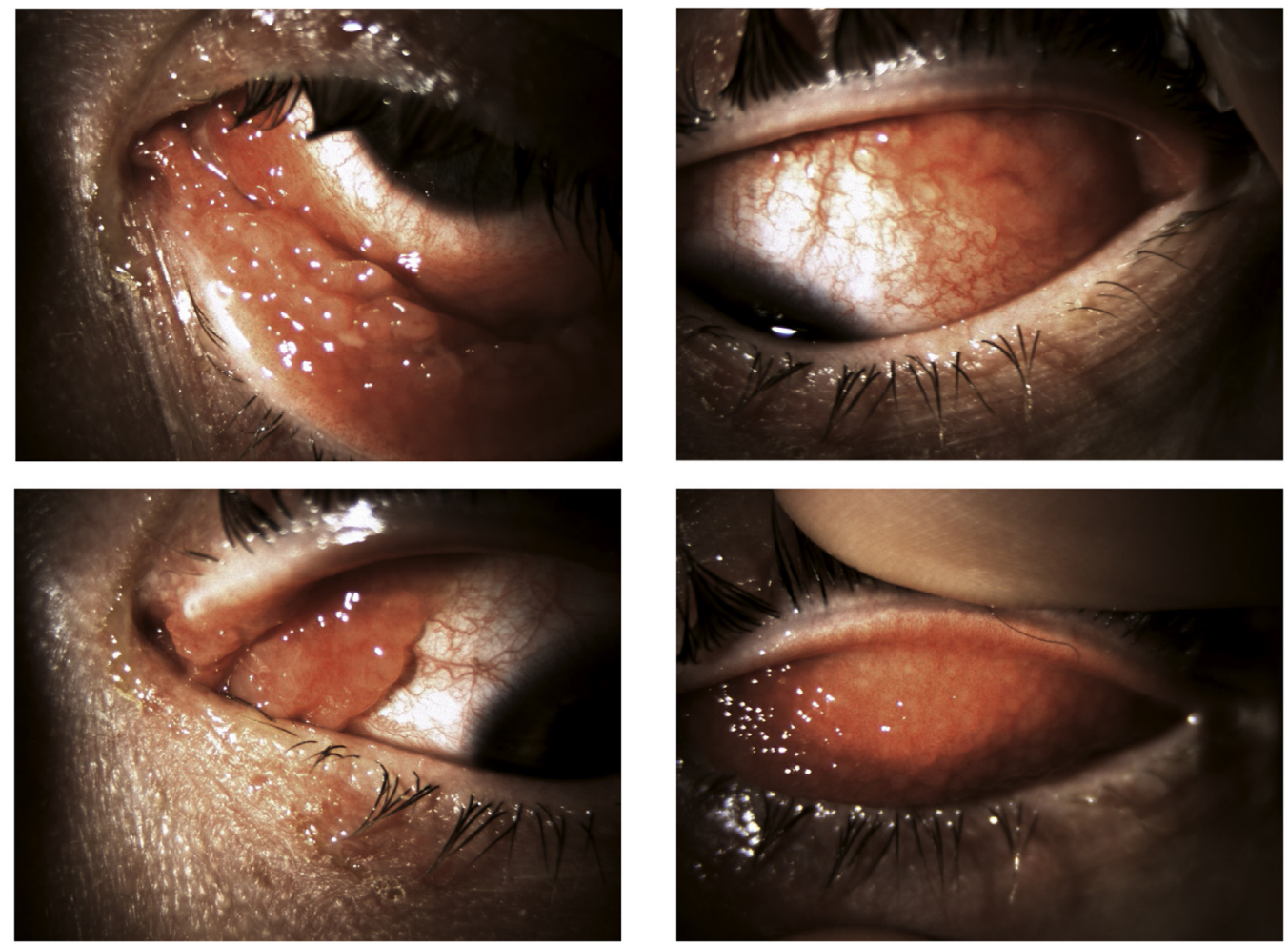

Figure 1. Follicules géants au niveau des conjonctives tarsales et bulbaires.

Une conjonctivite trainante et résistante aux traitements habituels est suspecte et doit pousser le clinicien à réaliser une PCR à la recherche de chlamydia trachomatis [4].

\section{Déclaration de liens d'intérêts}

Les auteurs déclarent ne pas avoir de liens d'intérêts.

\section{Références}

[1] Rapport Infections Sexuellement Transmissibles. Santé publique et Surveillance. Bruxelles, Belgique (๔ WIV-ISP, Bruxelles, 2016 Éditeur responsable : Dr Myriam Sneyers Dépôt légal : D/2016/2505/54); 2015.

[2] Creuzot-Garcher C. Les conjonctivites à inclusions de l'adulte. J Fr Ophtalmol 1999;22:577-80.

[3] Lanjouw E, Ouburg S, de Vries HJ, Stary A, Radcliffe K, Unemo M. 2015 European guideline on the management of Chlamydia trachomatis infections. Int J STD AIDS 2015 [OnlineFirst].

[4] Azari AA, Barney NP. Conjunctivitis: a systematic review of diagnosis and treatment. JAMA 2013;310:1721-9.

[5] Mohamed-Noriega K, Mohamed-Noriega J, Valdes-Navarro MA, Cuervo-Lozano EE, Fernandez-Espinosa MC, Mohamed-Hamsho J. Conjunctival infection with Chlamydia trachomatis in sexual partners of patients with adult inclusion conjunctivitis. Int Ophthalmol 2015;35:179-85.
[6] Mishori R, McClaskey EL, Winklerprins VJ. Chlamydia trachomatis infections: screening, diagnosis, and management. Am Fam Phys 2012;86:1-6.

[7] Postema EJ, Remeijer L, vander Meijden WI. Epidemiology of genital chlamydial infections in patients with chlamydial conjunctivitis; a retrospectivestudy. Genitourin Med 1996;72:203-5

[8] Martinez Weber C, Eichenbaum JW. Acute red eye. Postgrad Med 1997;101:1-9.

[9] Centers for Disease Control, Prevention. Sexually transmitted diseases treatment guidelines, 2010. MMWR Morb Mortal Wkly Rep 2010;59:44-55.

M. Terlinchamp*, B. Duchesne Service d'ophtalmologie, $\mathrm{CHU}$ de liège, avenue de L'Hòpital 1, 4000 Liège, Belgique

* Auteur correspondant.

Adresse e-mail : matthieu.terlinchamp@gmail.com (M. Terlinchamp) Disponible sur Internet le 18 novembre 2019

https://doi.org/10.1016/j.jfo.2019.03.036

0181-5512/ @ 2019 Elsevier Masson SAS. Tous droits réservés. 